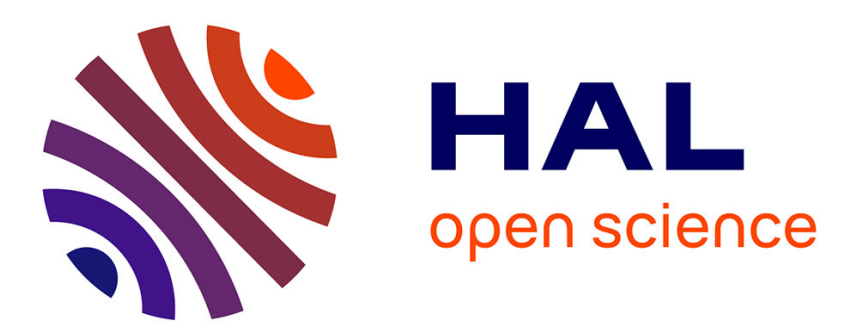

\title{
Dysprosium-Doped Silica Fiber as Saturable Absorber in a Mid-IR Fiber Laser
}

Pascal Paradis, Michèle Ude, Vincent Fortin, Bernard Dussardier, Stanislaw Trzesien, Réal Vallée, Martin Bernier

\section{- To cite this version:}

Pascal Paradis, Michèle Ude, Vincent Fortin, Bernard Dussardier, Stanislaw Trzesien, et al.. Dysprosium-Doped Silica Fiber as Saturable Absorber in a Mid-IR Fiber Laser. IEEE Photonics Conference, Oct 2021, (Virtuel), Canada. 10.1109/IPC48725.2021.9592883 . hal-03433128

\section{HAL Id: hal-03433128 \\ https://hal.science/hal-03433128}

Submitted on 17 Nov 2021

HAL is a multi-disciplinary open access archive for the deposit and dissemination of scientific research documents, whether they are published or not. The documents may come from teaching and research institutions in France or abroad, or from public or private research centers.
L'archive ouverte pluridisciplinaire HAL, est destinée au dépôt et à la diffusion de documents scientifiques de niveau recherche, publiés ou non, émanant des établissements d'enseignement et de recherche français ou étrangers, des laboratoires publics ou privés. 


\title{
Dysprosium-Doped Silica Fiber as Saturable Absorber in a Mid-IR Fiber Laser
}

\author{
Pascal Paradis \\ Center for Optics, Photonics and Lasers \\ Université Laval \\ Québec, Canada \\ pascal.paradis.2@ulaval.ca \\ Michèle Ude \\ Université de Nice Côte d'Azur, CNRS \\ Institut de Physique de Nice \\ Nice, France \\ michele.ude@unice.fr
}

\author{
Vincent Fortin \\ Center for Optics, Photonics and Lasers \\ Université Laval \\ Québec, Canada \\ vincent.fortin@copl.ulaval.ca \\ Bernard Dussardier \\ Université de Nice Côte d'Azur, CNRS \\ Institut de Physique de Nice \\ Nice, France \\ bernard.dussardier@unice.fr \\ Martin Bernier \\ Center for Optics, Photonics and Lasers \\ Université Laval \\ Québec, Canada \\ martin.bernier@copl.ulaval.ca
}

\author{
Stanislaw Trzesien \\ Université de Nice Côte d'Azur, CNRS \\ Institut de Physique de Nice \\ Nice, France \\ stanislaw.trzesien@unice.fr \\ Réal Vallée \\ Center for Optics, Photonics and Lasers \\ Université Laval \\ Québec, Canada \\ rvallee@copl.ulaval.ca
}

\begin{abstract}
We demonstrate the use of a dysprosium-doped silica fiber saturable absorber in a passively Q-switched erbium-doped fluoride fiber laser operating at $2.8 \mu \mathrm{m}$.
\end{abstract}

Keywords-fiber, laser, components, saturable absorber, Q-switched

\section{INTRODUCTION}

In recent years, mid-infrared (MIR) fiber lasers have attracted much interest from researchers of both academia and industries for their excellent beam quality and their power scalability as well as for their emission spectra between $2.5 \mu \mathrm{m}$ and $5 \mu \mathrm{m}$ [1], [2]. These inherent qualities make those fiber lasers quite promising for biomedical applications, material processing, remote sensing and defense and security applications.

Pulsed MIR lasers are of particular interest to cope with the demanding specifications for proper material processing, in particular for biomedical applications where material should be processed without damaging the surrounding tissues [2]. Pulsed fiber lasers are also used to generate supercontinua which can then be used for remote sensing of multiple atmospheric pollutants at once in the MIR atmospheric transmission window. Up to now, gain-switched, Q-switched, and mode-locked fiber lasers have all been demonstrated in the MIR using different methods and components. However, most of the reported systems are based on free space optics due to the lack of commercially available fiberized components optimized for MIR wavelengths, such as optical isolators, waveplates and saturable absorbers [1]. Among all those pulsed lasers, the gain-switched fiber laser was the only one that could be made all-fiber because its architecture can be simplified to a gain medium made of a rare earth-doped fluoride fiber bounded by two fiber Bragg gratings (FBGs) as the input and output couplers [3], the pulsing mechanism being based on the fast electronic modulation of the pump. All MIR Q-switched and mode-locked fiber lasers require either a saturable absorber that is not fiberized or an optical isolator and waveplates, or even all of these. Thus, the robustness required for field applications and long-term reliability when deployed in non-controlled environments is not achieved which is slowing down the adoption of these otherwise great lasers by other scientific communities for their specific applications.

In this paper, we present the first passively Q-switched fiber laser operating at $2791 \mathrm{~nm}$ based on a dysprosium-doped silica fiber as the saturable absorber, an erbium-doped fluoride fiber as the gain medium and an FBG as the output coupler. This demonstration is the first step towards achieving the first all-fiber Q-switched laser operating in the MIR.

\section{EXPERIMENTAL SETUP}

As presented in fig. 1, the laser cavity is made of a $2.5 \mathrm{~m}$-long $7 \mathrm{~mol} . \% \mathrm{Er}^{3+}$-doped fluoride fiber (Le Verre Fluoré) as the gain medium, a Dy ${ }^{3+}$-doped silica fiber made at the Institut de Physique de Nice as the fiberized saturable absorber, an FBG as the output coupler and a gold mirror as the high reflectivity mirror. The FBG has a peak reflectivity of $70 \%$ at $2791 \mathrm{~nm}$ with a FWHM of $3.0 \mathrm{~nm}$, and is written using a femtosecond pulses through the fiber's protective coating to preserve the original robustness of the fiber [4]. The gain fiber is backward pumped with a $976 \mathrm{~nm}$ pump diode (BWT, K976DB2RN-10.00W). To simplify the manipulation of the laser cavity while trying different saturable absorber lengths, the pump injection into the gain fiber and the high reflectivity mirror on the saturable absorber side are using free space optics. The aspherical ZnSe lenses used in the laser setup transmit around $95 \%$ of the signal at $2.8 \mu \mathrm{m}$ thanks to an anti-reflective coating optimized for this wavelength. The fluoride fiber tip at the output of the laser 




Fig. 2. Experimental setup of the Q-switched fiber laser based on the $\mathrm{Dy}^{3+}$-doped silica fiber. (M: gold mirror, DM: dichroic mirror, CMS: cladding mode stripper)

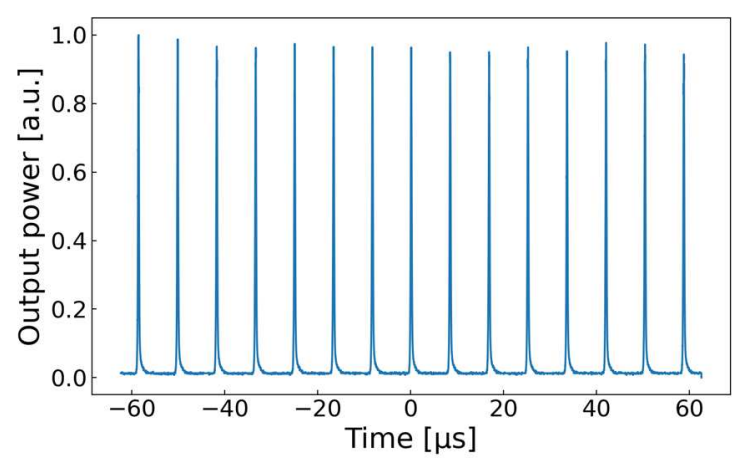

Fig. 1. Typical Q-switched pulse train generated by this laser cavity.

cavity is angle-cleaved at $6.5^{\circ}$ while the $\mathrm{Dy}^{3+}$-doped silica fiber is also angle-cleaved at around $5^{\circ}$ for each tested length to prevent parasitic feedback to the cavity. The dichroic mirror inside the laser cavity removes the residual pump power which can be significant at shorter silica fiber lengths and is also used when testing the same cavity with a semiconductor saturable absorber mirror (SESAM) for comparison [5]. The $\mathrm{Dy}^{3+}$-doped silica fiber is spliced directly to the $\mathrm{Er}^{3+}$-doped fluoride fiber to improve the stability of the setup and to ensure the same experimental conditions even when shortening the silica fiber. This single mode splice has a transmission of $78 \%$, which is not the best that could be achieved, but is more than enough to ensure an efficient Q-switching regime.

\section{RESULTS}

A typical pulse train with the same characteristics as any pulse train from any passively Q-switched laser cavity, as shown in fig. 2, can be generated by this fiber laser. It should be noted that no Q-switching is observed with the same laser setup without the $\mathrm{Dy}^{3+}$-doped silica fiber. A Q-switched regime is achieved from the threshold of the laser emission to the highest pump power available in this setup for lengths of Dy3+-doped silica fiber ranging from 2 to $110 \mathrm{~mm}$. Pulse durations are ranging from $250 \mathrm{~ns}$ to $2 \mu \mathrm{s}$ at repetition rates ranging from $20 \mathrm{kHz}$ to $250 \mathrm{kHz}$ depending on the length of saturable absorber and the pump power. The performances and behavior of this first Q-switched laser based on such fiber-based saturable absorber will be discussed. It should be noted that the damage threshold of such fiberized saturable absorber is much higher than for typical 2D saturable absorbers since the absorption is volumetric through the whole length of the doped fiber.

\section{CONCLUSION}

We report on a new fiberized MIR saturable absorber based on a $\mathrm{Dy}^{3+}$-doped silica fiber. This robust saturable absorber is shown efficient for passively Q-switching a fiber laser at $2.8 \mu \mathrm{m}$ based on an erbium-doped fluoride fiber laser as gain medium. During the presentation, the behavior and characteristics of this new saturable absorber and the performances of the laser cavity will be discussed in more details.

\section{REFERENCES}

[1] S. D. Jackson and R. K. Jain, "Fiber-based sources of coherent MIR radiation: key advances and future prospects (invited)," Opt. Express, vol. 28, no. 21, p. 30964, Oct. 2020

[2] X. Zhu et al., "Pulsed fluoride fiber lasers at $3 \mu \mathrm{m}$ [Invited]," J. Opt. Soc. Am. B, vol. 34, no. 3, p. A15, Mar. 2017

[3] P. Paradis, V. Fortin, Y. O. Aydin, R. Vallée, and M. Bernier, “10 W-level gain-switched all-fiber laser at 2.8 $\mu$ m," Opt. Lett., vol. 43, no. 13, p. 3196 , Jul. 2018

[4] J. Habel, T. Boilard, J.-S. Frenière, F. Trépanier, and M. Bernier, "Femtosecond FBG Written through the Coating for Sensing Applications," Sensors, vol. 17, no. 11 , p. 2519, Nov. 2017

[5] P. Paradis, V. Fortin, B. Dussardier, R. Vallée, and M. Bernier, "Towards Mid-IR Pulsed All-Fiber Lasers Based on Saturable Absorbers," in 2020 IEEE Photonics Conference (IPC), 2020, pp. 1-2 